\title{
COMONOTONIC MEASURES OF MULTIVARIATE RISKS
}

\author{
IVAR EKELAND $^{\dagger}$ ALFRED GALICHON $^{\S}$ MARC HENRY M $^{\ddagger}$
}

\begin{abstract}
We propose a multivariate extension of a well-known characterization by S. Kusuoka of regular and coherent risk measures as maximal correlation functionals. This involves an extension of the notion of comonotonicity to random vectors through generalized quantile functions. Moreover, we propose to replace the current law invariance, subadditivity and comonotonicity axioms by an equivalent property we call strong coherence and that we argue has more natural economic interpretation. Finally, we reformulate the computation of regular and coherent risk measures as an optimal transportation problem, for which we provide an algorithm and implementation.
\end{abstract}

Keywords: regular risk measures, coherent risk measures, comonotonicity, maximal correlation, optimal transportation, strongly coherent risk measures.

MSC 2000 subject classification: 91B06, 91B30, 90C08

Date: First version is October 16, 2007. The present version is of November 1, 2009. The authors thank two anonymous referees and an associate Editor who helped improving the paper. The authors are grateful to Guillaume Carlier, Rose-Anne Dana, Nicole El Karoui, Jean-Charles Rochet, Ludger Rüschendorf, and Nizar Touzi, as well as participants to the workshop "Multivariate and Dynamic Risk Measures" (IHP, Paris, October 2008), the SAS seminar at Princeton University, the Statistics Department Risk seminar at Columbia University for helpful discussions and comments. Galichon and Ekeland gratefully acknowledge support from Chaire EDF-Calyon "Finance and Développement Durable" and FiME, Laboratoire de Finance des Marchés de l'Energie (www.fime-lab.org), and Galichon that of Chaire Société Générale "Risques Financiers" and Chaire Axa "Assurance et Risques Majeurs". 


\section{INTRODUCTION}

The notion of coherent risk measure was proposed by Artzner, Delbaen, Eber and Heath in [1] as a set of axioms to be verified by a real-valued measure of the riskiness of an exposure. In addition to monotonicity, positive homogeneity and translation invariance, the proposed coherency axioms include subadditivity, which is loosely associated with hedging. Given this interpretation, it is natural to require the risk measure to be additive on the subsets of risky exposures that are comonotonic, as this situation corresponds to the worse-case scenario for the correlation of the risks. In [15], Kusuoka showed the remarkable result that law invariant coherent risk measures that are also comonotonic additive are defined by the integral of the quantile function with respect to a positive measure, a family that includes Expected Shortfall (also known as Conditional Value at Risk, or Expected Tail Loss).

The main drawback of this formulation is that it does not properly handle the case when the numéraires in which the risky payoffs are labeled are not perfect substitutes. This situation is commonly met in Finance. In a two-country economy with floating exchange rates, the fact that claims on payoffs in different currencies are not perfectly substitutable is known as the Siegel paradox; in the study of the term structure of interest rates, the fact that various maturities are (not) perfect substitutes is called the (failure of the) pure expectation hypothesis. The technical difficulty impeding a generalization to the case of a multivariate risk measure is that the traditional definition of comonotonicity relies on the order in $\mathbb{R}$. When dealing with portfolios of risk that are non perfectly substituable, as Jouini, Meddeb and Touzi did in [13] for coherent risk measures, and Rüschendorf in [17] for law invariant convex risk measures, the right notion of multivariate comonotonicity is not immediately apparent.

The present work circumvents these drawbacks to generalize Kusuoka's result to multivariate risk portfolios, and proposes a simplifying reformulation of the axioms with firm decision theoretic foundations. First, we propose an alternative axiom called strong coherence, which is equivalent to the axioms in [15] and which, unlike the latter, extends to the multivariate setting. We then make use of a variational characterization of Kusuoka's axioms and representation in order to generalize his results to the multivariate case. We show 
that multivariate risk measures that satisfy strong coherence have the same representation as in [15], which we discuss further below.

The work is organized as follows. The first section motivates a new notion called strong coherence which is shown to be intimately related to existing risk measures axioms, yet appears to be more natural. The second section shows how the concept of comonotonic regular risk measures can be extended to the case of multivariate risks, by introducing a proper generalization of the notion of comonotonicity and giving a representation theorem. The third section discusses in depth the relation with Optimal Transportation Theory, and shows important examples of actual computations.

Notations and conventions. Let $(\Omega, \mathcal{F}, \mathbb{P})$ be a probability space, which is standard in the terminology of [14], that is $\mathbb{P}$ is nonatomic and $L^{2}(\Omega, \mathcal{F}, \mathbb{P})$ is separable. Let $X: \Omega \rightarrow \mathbb{R}^{d}$ be a random vector; we denote the distribution law of $X$ by $\mathcal{L}_{X}$, hence $\mathcal{L}_{X}=X \# \mathbb{P}$, where $X \# \mathbb{P}:=\mathbb{P} X^{-1}$ denotes the push-forward of probability measure $\mathbb{P}$ by $X$. The equidistribution class of $X$ is the set of random vectors with distribution with respect to $\mathbb{P}$ equal to $\mathcal{L}_{X}$ (reference to $\mathbb{P}$ will be implicit unless stated otherwise). As explained in the appendix, essentially one element in the equidistribution class of $X$ has the property of being the gradient of a convex function; this random element is called the (generalized) quantile function associated with the distribution $\mathcal{L}_{X}$ and denoted by $Q_{X}$ (in dimension 1 , this is the quantile function of distribution $\mathcal{L}_{X}$ in the usual sense). We denote by $\mathcal{M}\left(\mathcal{L}, \mathcal{L}^{\prime}\right)$ the set of probability measures on $\mathbb{R}^{d} \times \mathbb{R}^{d}$ with marginals $\mathcal{L}$ and $\mathcal{L}^{\prime}$. We call $L_{d}^{2}(\mathbb{P})$ (abbreviated in $\left.L_{d}^{2}\right)$ the equivalence class of $\mathcal{F}$-measurable functions $\Omega \rightarrow \mathbb{R}^{d}$ with a finite second moment modulo $\mathbb{P}$-negligible events. We call $\mathcal{P}_{2}\left(\mathbb{R}^{d}\right)$ the set of probability on $\mathbb{R}^{d}$ with finite second moment. Finally, for two elements $X, Y$ of $L_{d}^{2}$, we write $X \sim Y$ to indicate equality in distribution, that is $\mathcal{L}_{X}=\mathcal{L}_{Y}$. We also write $X \sim \mathcal{L}_{X}$. Define c.l.s.c. $\left(\mathbb{R}^{d}\right)$ as the class of convex lower semi-continuous functions on $\mathbb{R}^{d}$, and the Legendre-Fenchel conjugate of $V \in$ c.l.s.c. $\left(\mathbb{R}^{d}\right)$ as $V^{*}(x)=\sup _{y \in \mathbb{R}^{d}}[x \cdot y-V(y)]$. In all that follows, the dot "." will denote the standard scalar product in $\mathbb{R}^{d}$ or $L_{d}^{2} \cdot \mathcal{M}_{d}(\mathbb{R})$ denotes the set of $d \times d$ matrices, and $\mathcal{O}_{d}(\mathbb{R})$ the orthogonal group in dimension $d$. For $M \in \mathcal{M}_{d}(\mathbb{R}), M^{T}$ denotes the matrix transpose of $M$. For a function $V: \mathbb{R}^{d} \rightarrow \mathbb{R}$ differentiable at $x$, we denote $\nabla V(x)$ the 
gradient of $V$ at $x$; this is the vector $\left(\frac{\partial V(x)}{\partial x_{1}}, \ldots, \frac{\partial V(x)}{\partial x_{d}}\right) \in \mathbb{R}^{d}$. When $V$ is twice differentiable at $x$, we denote $D^{2} V$ the Hessian matrix of $V$ that is the matrix $\left(\frac{\partial^{2} V(x)}{\partial x_{i} \partial x_{j}}\right)_{1 \leq i, j \leq d}$. By Aleksandrov's theorem, a convex function is (Lebesgue-) almost everywhere differentiable on the interior of its domain (see [22], pp. 58-59), so $\nabla V$ and $D^{2} V$ exist almost everywhere. For a functional $\Phi$ defined on a Banach space, we denote $D \Phi$ its Fréchet derivative.

\section{Strong COHEREnCE: A NATURAL AXIOMATiC CHARACTERIZATion}

In this section we advocate a very simple axiomatic setting, called strong coherence which will be shown to be equivalent to the more classical axiomatic framework described in the next section. We argue that this axiom has more intuitive appeal than the classical (equivalent) axioms.

1.1. Motivation: Structure Neutrality. The regulating instances of the banking industry are confronted with the problem of imposing rules to the banks to determine the amount of regulatory capital they should budget to cover their risky exposure. A notable example of such a rule is the Value-at-Risk, imposed by the Basel II committee, but a number of competing rules have been proposed. We call $X \in L_{d}^{2}$ the vector of random $\operatorname{losses}^{1}$ of a given bank. Note that contrary to a convention often adopted in the literature, we chose to account positively for net losses: $X$ is a vector of effective losses. Also note that we have supposed that the risk is multivariate, which means that there are multiple numéraires, which, depending on the nature of the problem, can be several assets, several term maturities, or several non-monetary risks of different nature. We suppose that these multiple numéraires cannot be easily exchanged into one another: the problem is intrinsiquely multivariate. This could be the case if the firm (or the regulator) is unable or unwilling to define a monetary equivalent for the various dimensions of its risks. For instance, an oil company is likely to be unable to estimate a dollar amount to price its environmental risk; similarly,

\footnotetext{
${ }^{1}$ In this paper we have chosen to restrict ourselves to the case where risks are in $L^{2}\left(\mathbb{R}^{d}\right)$ for notational convenience, but all results in the paper carry without difficulty over to the case where the risks are in in $L^{p}\left(\mathbb{R}^{d}\right)$ for $p \in(1,+\infty)$.
} 
a pharmaceutical company may be unwilling to give a monetary estimate for the health hazard its product carry.

To a random vector of losses $X$ one associates a number $\varrho(X)$ which measures the intensity of the risk incurred. The unit in which $\varrho$ is to be thought of as some extra currency unity, or alternatively a non-monetary score; it is not assumed to be one of the monetary units associated with the various dimensions of the vector of the risks. This score is used by investors to compare the risks of two companies, or by regulators to set limits to risky exposures for regulated firms. An important desirable feature of the rule proposed by the regulator is to avoid regulatory arbitrage. Here, a regulatory arbitrage would be possible if the firms could split their risk into several different subsidiaries $S_{i}, i=1, \ldots, N$ with independent legal existence, so that the the shareholder's economic risk remained the same $X=X_{1}+\ldots+X_{N}$, but such that the amount of the shareholder's capital which is required to be budgeted to cover their risk were strictly inferior after the split, namely such that $\varrho(X)>\varrho\left(X_{1}\right)+\ldots+\varrho\left(X_{N}\right)$. To avoid this, we shall impose the requirement of subadditivity, that is

$$
\varrho\left(X_{1}+\ldots+X_{N}\right) \leq \varrho\left(X_{1}\right)+\ldots+\varrho\left(X_{N}\right)
$$

for all possible dependent risk exposures $\left(X_{1}, \ldots, X_{N}\right) \in\left(L_{d}^{2}\right)^{N}$. We now argue that the regulator is only interested in the amount and the intensity of the risk, not in its operational nature: the capital budgeted should be the same for a contingent loss of $1 \%$ of the total capital at risk no matter how the loss occurred (whether on the foreign exchange market, the stock market, the credit market, etc.) This translates mathematically into the requirement that the regulatory capital to budget should only depend on the distribution of the risk $X$, that is, the rule should satisfy the law invariance property:

Definition 1. A functional $\varrho: L^{2} \rightarrow \mathbb{R}$ is called law-invariant if $\varrho(X)=\varrho(Y)$ when $X \sim Y$, where $\sim$ denotes equality in distribution.

By combining together subadditivity and law invariance, we get the natural requirement for the capital budgeting rule, that $\varrho\left(\tilde{X}_{1}+\ldots+\tilde{X}_{N}\right) \leq \varrho\left(X_{1}\right)+\ldots+\varrho\left(X_{N}\right)$ for all $X, \tilde{X}$ in $\left(L_{d}^{2}\right)^{N}$ such that $X_{i} \sim \tilde{X}_{i}$ for all $i=1, \ldots, N$. However, in order to prevent giving a premium to conglomerates, and to avoid imposing an overconservative rule to the regulated firms, 
one is led to impose the inequality to be sharp and pose the structure neutrality axiom

$$
\varrho\left(X_{1}\right)+\ldots+\varrho\left(X_{N}\right)=\sup _{\tilde{X}_{i} \sim X_{i}} \varrho\left(\tilde{X}_{1}+\ldots+\tilde{X}_{N}\right)
$$

This requirement is notably failed by the Value-at-Risk, which leads to the fact that the Value-at-Risk as a capital budgeting rule is not neutral to the structure of the firm. This result should be read in the perspective of the corporate finance literature on the optimal structure of the firm, originating in the celebrated Modigliani-Miller theorem, according to which the value of the firm does not depend on the structure of its capital. This point is explained in detail in [12], where an explicit construction is provided. We introduce the axiom of strong coherence to be satisfied by a measure of the riskiness of a portfolio of risk exposures (potential losses) $X \in L_{d}^{2}$.

Definition 2 (Strong coherence). For $\mu \in \mathcal{P}_{2}\left(\mathbb{R}^{d}\right)$, a functional $\varrho: L_{d}^{2} \rightarrow \mathbb{R}$ is called a strongly coherent risk measure if (i) it is convex continuous, and (ii) it is structure neutral: for all $X, Y \in \mathcal{L}_{d}^{2}$,

$$
\varrho(X)+\varrho(Y)=\sup \{\varrho(\tilde{X}+\tilde{Y}): \tilde{X} \sim X ; \tilde{Y} \sim Y\}
$$

The convexity axiom can be justified by a risk aversion principle: in general, one should prefer to diversify risk. The structure neutrality axiom, being defined as a supremum over all correlation structures, can be interpreted as a provision against worst-case scenarios, and may be seen as unduly conservative. However, this axiom is no more conservative than the set of axioms defining a regular coherent risk measure as we shall see.

As we shall see also, strongly coherent risk measures satisfy all the classical axioms of coherent risk measures (recalled in definition 4 below) let alone monotonicity, for which the multivariate extension is not obvious. In particular, these measures satisfy positive homogeneity and translation invariance. They also satisfy law invariance, which can be seen by taking $Y=0$ in the definition above.

We now show that strongly coherent risk measures are represented by maximal correlation functionals with respect to a given random vector or scenario. 
1.2. Characterization of strongly coherent risk measures. We are now going to show that the strong coherence property essentially characterizes a class of risk measures known as maximal correlation risk measures, which we shall first recall the definition of.

1.2.1. Maximal correlation measures. We first define maximal correlation risk measures (in the terminology of Rüschendorf who introduced them in the multivariate case, see e.g. [17]). These measures will generalize the variational formulation for coherent regular risk measures given in (2.1) below.

Definition 3 (Maximal correlation measures). A functional $\varrho_{\mu}: L_{d}^{2} \rightarrow \mathbb{R}$ is called a maximal correlation risk measure with respect to a baseline distribution $\mu$ if for all $X \in L_{d}^{2}$,

$$
\varrho_{\mu}(X):=\sup \{\mathbb{E}[X \cdot \tilde{U}]: \tilde{U} \sim \mu\} .
$$

Our notion of maximal correlation risk measure is essentially the same as Rüschendorf's, with a few minor variants: Rüschendorf defines his measures on $L_{d}^{\infty}$ instead of $L_{d}^{2}$, and imposes the extra requirements $U_{i} \geq 0$ and $E_{\mu}\left[U_{i}\right]=1$ for $i=1, \ldots, d$, which we do not impose for now.

Remark 1 (Geometric interpretation). The maximum correlation measure with respect to measure $\mu$ is the support function of the equidistribution class of $\mu$.

Example 1 (Multivariate Expected Shortfall). An interesting example of univariate risk measure within the class of maximal correlation risk measures is the expected shortfall, also known as conditional value at risk. This risk measure can be generalized to the multivariate setting by defining the $\alpha$-expected shortfall of a risk exposure $X$ as the maximal correlation measure when the baseline risk $U$ is a Bernoulli random vector (i.e. its distribution $\mathcal{L}_{U}$ is determined by $U=(1 / \alpha, \ldots, 1 / \alpha)^{T}$ with probability $\alpha$ and 0 with probability $1-\alpha)$. In such case, one can easily check that if $\mathcal{L}_{X}$ is absolutely continuous, then defining $W(x)=\max \left(\sum_{i=1}^{d} x_{i}-c, 0\right)$, with $c$ given by requirement $\operatorname{Pr}\left(\sum_{i=1}^{d} x_{i} \geq c\right)=\alpha$, it follows that $W$ is convex and $\nabla W$ exists $\mathcal{L}_{X}$ almost everywhere and pushes $\mathcal{L}_{X}$ to $\mathcal{L}_{U}$ as in proposition 7 ; therefore the maximal correlation measure is given in this case by 
$E\left[\left(\sum_{i=1}^{d} X_{i}\right) 1\left\{\sum_{i=1}^{d} X_{i} \geq c\right\}\right]$. In other words, the maximum correlation measure in this example is the (univariate) $\alpha$-expected shortfall for $Y=\sum_{i=1}^{d} X_{i}$.

Example 2. With a more complex baseline risk, other important examples where explicit or numerical computation is possible include the cases when 1) the baseline risk and the risk to be measured are both Gaussian, or 2) the baseline risk is uniform on $[0,1]^{d}$ and the risk to be measured has a discrete distribution. Both these cases are treated in detail in Section 4.

Let us first recall the following lemma, which emphasizes the symmetry between the roles played by the equivalence class of $X$ and $U$ in the definition above.

Lemma 1. For any choice of $U \sim \mu$, with $\mu \in \mathcal{P}_{2}\left(\mathbb{R}^{d}\right)$, one has

$$
\varrho_{\mu}(X)=\sup \{\mathbb{E}[\tilde{X} \cdot U]: \tilde{X} \sim X\}
$$

and $U$ is called the baseline risk associated with $\varrho_{\mu}$. It follows that $\varrho_{\mu}$ is law invariant.

Proof. See (2.12) in [17].

1.2.2. Characterization. We now turn to our first main result, which is a characterization of strongly coherent risk measures. We first prove a useful intermediate characterization in proposition 1 below. We shall use Lemma A.4 from [14], which we quote here for the reader's convenience. Denote by $\mathcal{A}$ the set of bimeasurable bijections $\sigma$ from $(\Omega, \mathcal{A}, \mathbb{P})$ into itself which preserve the probability, so that $\sigma \# \mathbb{P}=\mathbb{P}$. Recall that $(\Omega, \mathcal{F}, \mathbb{P})$ was assumed to be a probability space which does not have atoms, and such that $L^{2}(\Omega, \mathcal{F}, \mathbb{P})$ is separable.

Lemma 2. Let $C$ be a norm closed subset of $L^{2}(\Omega, \mathcal{F}, \mathbb{P})$. Then the following are equivalent:

(1) $C$ is law invariant, that is $X \in C$ and $X \sim Y$ implies that $Y \in C$

(2) $C$ is transformation invariant, that is for any $X \in C$ and any $\sigma \in \mathcal{A}$, we have $X \circ \sigma \in C$

As an immediate consequence, we have the following result:

Proposition 1. A convex continuous functional $\varrho: L_{d}^{2} \rightarrow \mathbb{R}$ is a strongly coherent risk measure if and only if we have:

$$
\varrho(X)+\varrho(Y)=\sup \{\varrho(X \circ \sigma+Y \circ \tau): \sigma, \tau \in \mathcal{A}\} .
$$


Proof. Clearly $X \circ \sigma \sim X$ and $Y \circ \tau \sim Y$. Hence:

$$
\sup \{\varrho(X \circ \sigma+Y \circ \tau): \sigma, \tau \in \mathcal{A}\} \leq \sup \{\varrho(\tilde{X}+\tilde{Y}): \tilde{X} \sim X, \tilde{Y} \sim Y\}
$$

To prove the converse, take any $\varepsilon>0$ and some $X^{\prime} \sim X$ and $Y^{\prime} \sim Y$ such that:

$$
\varrho\left(X^{\prime}+Y^{\prime}\right) \geq \sup \{\varrho(\tilde{X}+\tilde{Y}): \tilde{X} \sim X, \tilde{Y} \sim Y\}-\varepsilon
$$

Consider the set $\{X \circ \sigma: \sigma \in \mathcal{A}\}$ and denote by $C$ its closure in $L^{2}$. It is obviously transformation invariant. By the preceding Lemma, it is also law invariant. Since $X \in C$ and $X^{\prime} \sim X$, we must have $X^{\prime} \in C$, meaning that there exists a sequence $\sigma_{n} \in \mathcal{A}$ with $\left\|X \circ \sigma_{n}-X^{\prime}\right\| \longrightarrow 0$. Similarly, there must exist a sequence $\tau_{n} \in \mathcal{A}$ with $\left\|Y \circ \tau_{n}-Y^{\prime}\right\| \longrightarrow$ 0 . Since $\varrho$ is continuous, it follows that, for $n$ large enough, we have:

$$
\begin{aligned}
\sup \{\varrho(X \circ \sigma+Y \circ \tau): \sigma, \tau \in \mathcal{A}\} & \geq \varrho\left(X \circ \sigma_{n}+Y \circ \tau_{n}\right) \geq \varrho\left(X^{\prime}+Y^{\prime}\right)-\varepsilon \\
& \geq \sup \{\varrho(\tilde{X}+\tilde{Y}): \tilde{X} \sim X, \tilde{Y} \sim Y\}-2 \varepsilon
\end{aligned}
$$

and since this holds for any $\varepsilon>0$, the converse of (1.2) holds

We can now state our main result:

Theorem 1. Let $(\Omega, \mathcal{F}, \mathbb{P})$ be a probability space which does not have atoms, and such that $L^{2}(\Omega, \mathcal{F}, \mathbb{P})$ is separable. Let $\varrho$ be a functional defined on $L_{d}^{2}$. Then the following propositions are equivalent:

(i): $\varrho$ is a strongly coherent risk measure;

(ii): $\varrho$ is a maximal correlation risk measure

Before we turn to the proof, note that this representation implies immediately that strongly coherent risk measures are in particular positive homogenous and translation invariant, as announced above.

Proof. We first show (i) $\Rightarrow$ (ii). As the proof is quite long, we will punctuate it with several lemmas. 
By the preceding proposition and law invariance, it is enough to prove that:

$$
\varrho(X)+\varrho(Y)=\sup _{\sigma \in \mathcal{A}} \varrho(X+Y \circ \sigma)
$$

Call $\varrho^{*}$ the Legendre transform of $\varrho$ in $L_{d}^{2}$.

Lemma 3. $\varrho^{*}$ is law-invariant.

Proof. For $\sigma \in \mathcal{A}$, one has $\varrho^{*}\left(X^{*} \circ \sigma\right)=\sup _{X \in L_{d}^{2}}\left\{\left\langle X^{*} \circ \sigma, X\right\rangle-\varrho(X)\right\}$, so $\varrho^{*}\left(X^{*} \circ \sigma\right)=$ $\sup _{X \in L_{d}^{2}}\left\{\left\langle X^{*}, X \circ \sigma^{-1}\right\rangle-\varrho(X)\right\}=\sup _{X \in L_{d}^{2}}\left\{\left\langle X^{*}, X \circ \sigma^{-1}\right\rangle-\varrho\left(X \circ \sigma^{-1}\right)\right\}=\varrho^{*}\left(X^{*}\right)$.

Lemma 4. If the functions $f_{i}, i \in I$, are l.s.c. convex functions, then

$$
\left(\sup _{i} f_{i}\right)^{*}=\left(\inf _{i} f_{i}^{*}\right)^{* *}
$$

Proof. For a given l.s.c. convex function $f, f \leq\left(\sup _{i} f_{i}\right)^{*}$ is equivalent to $f^{*} \geq \sup _{i} f_{i}$, hence to $f \geq f_{i}$ for all $i$, hence to $f^{*} \leq f_{i}^{*}$ for all $i$, hence $f \leq \inf _{i} f_{i}^{*}$, hence, as $f$ is l.s.c. convex, to $f \leq\left(\inf _{i} f_{i}^{*}\right)^{* *}$, QED.

Applying lemma 4 to the structure neutrality equation, one has

$$
\begin{aligned}
\varrho^{*}\left(X^{*}\right)+\varrho^{*}\left(Y^{*}\right) & =\left(\inf _{\sigma \in \mathcal{A}} \sup _{X, Y}\left\{\left\langle X, X^{*}\right\rangle+\left\langle Y, Y^{*}\right\rangle-\varrho(X+Y \circ \sigma)\right\}\right)^{* *} \\
& =\left(\inf _{\sigma \in \mathcal{A}} \sup _{Y}\left\{\left\langle Y, Y^{*}\right\rangle+\varrho^{*}\left(X^{*}\right)-\left\langle Y \circ \sigma, X^{*}\right\rangle\right\}\right)^{* *} \\
& =\left(\varrho^{*}\left(X^{*}\right)+\inf _{\sigma \in \mathcal{A}} \sup _{Y}\left\langle Y, Y^{*}-X^{*} \circ \sigma^{-1}\right\rangle\right)^{* *} .
\end{aligned}
$$

The term in $\sup _{Y}(\ldots)$ on the right-hand side is 0 if $Y^{*}=X^{*} \circ \sigma^{-1}$ and $+\infty$ otherwise. Hence the previous formula becomes

$$
\varrho^{*}\left(X^{*}\right)+\varrho^{*}\left(Y^{*}\right)=\varphi^{* *}\left(X^{*}, Y^{*}\right)
$$

where we have defined

$$
\varphi\left(X^{*}, Y^{*}\right)=\begin{aligned}
& \varrho^{*}\left(X^{*}\right) \text { if } X^{*} \sim Y^{*} \\
& +\infty \text { otherwise. }
\end{aligned}
$$


Now suppose $\varphi\left(X^{*}, Y^{*}\right)<\infty$, hence that $\varrho^{*}\left(X^{*}\right)=\varrho^{*}\left(Y^{*}\right)<\infty$ and $X^{*} \sim Y^{*}$. As $\varphi \geq \varphi^{* *}$, it follows that $\varrho^{*}\left(X^{*}\right) \geq \varrho^{*}\left(X^{*}\right)+\varrho^{*}\left(Y^{*}\right)$ hence $\varrho^{*}\left(Y^{*}\right)=\varrho^{*}\left(X^{*}\right) \leq 0$, and $\varphi\left(X^{*}, Y^{*}\right) \leq 0$.

Suppose $\varphi\left(X^{*}, Y^{*}\right)<\infty$ and $\varphi\left(X^{*}, Y^{*}\right)-\varphi^{* *}\left(X^{*}, Y^{*}\right)<\varepsilon$. Replacing in (1.4), one finds that

$$
0 \leq-\varrho^{*}\left(X^{*}\right)=-\varrho^{*}\left(Y^{*}\right) \leq \varepsilon
$$

Lemma 5. $\varphi^{* *}$ is valued into $\{0,+\infty\}$.

Proof. As $\varphi^{*}=\varphi^{* * *}$, one has

$$
\begin{aligned}
\varphi^{*}(X, Y) & =\sup _{\left(X^{*}, Y^{*}\right)}\left\{\left\langle X, X^{*}\right\rangle+\left\langle Y, Y^{*}\right\rangle-\varphi^{* *}\left(X^{*}, Y^{*}\right)\right\} \\
& =\sup _{\left(X^{*}, Y^{*}\right)}\left\{\left\langle X, X^{*}\right\rangle+\left\langle Y, Y^{*}\right\rangle-\varphi\left(X^{*}, Y^{*}\right)\right\} .
\end{aligned}
$$

Taking a maximizing sequence $\left(X_{n}^{*}, Y_{n}^{*}\right)$ in the latter expression, one has necessarily $\varphi\left(X_{n}^{*}, Y_{n}^{*}\right)-$ $\varphi^{* *}\left(X_{n}^{*}, Y_{n}^{*}\right) \longrightarrow 0$. From the previous remark, $\varrho^{*}\left(X_{n}^{*}\right)=\varrho^{*}\left(Y_{n}^{*}\right) \longrightarrow 0$, hence $\varphi\left(X_{n}^{*}, Y_{n}^{*}\right) \longrightarrow$ 0 . Therefore

$$
\varphi^{*}(X, Y)=\sup _{\left(X^{*}, Y^{*}\right): \varphi\left(X^{*}, Y^{*}\right)=0}\left\{\left\langle X, X^{*}\right\rangle+\left\langle Y, Y^{*}\right\rangle\right\}
$$

which is clearly positively homogeneous of degree 1 . Its Legendre transform $\varphi^{* *}$ can therefore only take values 0 and $+\infty$, QED.

Therefore, there is a closed convex set $K$ such that $\varphi^{* *}$ is the indicator function of $K$, that is

$$
\varphi^{* *}\left(X^{*}, Y^{*}\right)=\begin{gathered}
0 \text { if }\left(X^{*}, Y^{*}\right) \in K \\
+\infty \text { otherwise }
\end{gathered}
$$

and condition (1.4) implies that

$$
\varrho^{*}\left(X^{*}\right)+\varrho^{*}\left(Y^{*}\right)=\begin{gathered}
0 \text { if }\left(X^{*}, Y^{*}\right) \in K \\
+\infty \text { otherwise }
\end{gathered}
$$

Note that if $\varrho^{*}\left(X^{*}\right)<\infty$, then $\varphi\left(X^{*}, Y^{*}\right)=\varrho^{*}\left(X^{*}\right)$ for all $Y^{*} \sim X^{*}$, and then $\varphi^{* *}\left(X^{*}, Y^{*}\right) \leq \varphi\left(X^{*}, Y^{*}\right)<\infty$. This implies that $\varphi^{* *}\left(X^{*}, Y^{*}\right)=0$, hence that $\varrho^{*}\left(X^{*}\right)=$ 
0. Therefore $\varrho^{*}$ is also an indicator function: there exists a closed convex set $C$ such that

$$
\varrho^{*}\left(X^{*}\right)=\begin{gathered}
0 \text { if } X^{*} \in C \\
+\infty \text { otherwise }
\end{gathered}
$$

By comparison of (1.7) and (1.8), one finds that

$$
K=C \times C
$$

By duality, (1.8) becomes

$$
\begin{aligned}
\varrho(X) & =\sup _{X^{*} \in C}\left\langle X^{*}, X\right\rangle \\
C & =\left\{X^{*} \mid \varrho^{*}\left(X^{*}\right)=0\right\}
\end{aligned}
$$

Condition (1.5) then implies that $\varphi$ is an indicator function: there exists a set $K_{0}$ (in general, neither a closed nor a convex set) such that

$$
\varphi\left(X^{*}, Y^{*}\right)=\begin{gathered}
0 \text { if }\left(X^{*}, Y^{*}\right) \in K_{0} \\
+\infty \text { otherwise }
\end{gathered}
$$

By comparison with formulas (1.5) and (1.6), one finds that

$$
\begin{aligned}
\left(X^{*}, Y^{*}\right) & \in K_{0} \Longleftrightarrow X^{*} \in C, Y^{*} \in C \text { and } X^{*} \sim Y^{*} \\
K & =\overline{c o} K_{0}
\end{aligned}
$$

Lemma 6. Denote by $\mathcal{E}(C)$ the set of strongly exposed points of $C$, and $\overline{K_{0}}$ the closure of $K_{0}$ for the norm topology in $L^{2} \times L^{2}$. Then

$$
\mathcal{E}(C) \times \mathcal{E}(C) \subset \overline{K_{0}}
$$

Proof. Recall (cf. [9]) that $X^{*}$ is strongly exposed in $C$ if there is a continuous linear form $X$ such that any maximizing sequence for $X$ in $C$ converges strongly to $X^{*}$ :

$$
\left.\begin{array}{c}
X_{n}^{*} \in C \\
\left\langle X, X_{n}^{*}\right\rangle \longrightarrow \sup _{C}\left\langle X, X^{*}\right\rangle
\end{array}\right\} \Longrightarrow\left\|X_{n}^{*}-X^{*}\right\| \longrightarrow 0
$$


For $\varepsilon>0$, denote by $T_{C}(X, \varepsilon)$ the set of $Y^{*} \in C$ such that $\sup _{Z^{*} \in C}\left\langle X, Z^{*}\right\rangle-\left\langle X, Y^{*}\right\rangle \leq \varepsilon$. Then $X^{*} \in C$ is strongly exposed by $X$ if and only if $\sup _{Z^{*} \in C}\left\langle X, Z^{*}\right\rangle=\left\langle X, X^{*}\right\rangle$ and $\delta\left[T_{C}(X, \varepsilon)\right]$ tends to 0 when $\varepsilon \longrightarrow 0$, where $\delta$ denotes the diameter,

$$
\delta\left[T_{C}(X, \varepsilon)\right]:=\sup \left\{\left\|X_{1}^{*}-X_{2}^{*}\right\| \mid X_{1}^{*} \in T_{C}(X, \varepsilon), X_{2}^{*} \in T_{C}(X, \varepsilon)\right\} .
$$

Going back to the problem, it is clear that if $X^{*}$ and $Y^{*}$ are strongly exposed in $C$, then $\left(X^{*}, Y^{*}\right)$ is strongly exposed in $C \times C$ :

$$
\mathcal{E}(C) \times \mathcal{E}(C) \subset \mathcal{E}(C \times C)=\mathcal{E}(K)
$$

We claim that every strongly exposed point of $K$ necessarily belongs to $\overline{K_{0}}$ (the closure is still the norm closure). Indeed, suppose there exists $\left(X_{1}^{*}, X_{2}^{*}\right) \in \mathcal{E}(K) \operatorname{such}$ that $\left(X_{1}^{*}, X_{2}^{*}\right) \notin$ $\overline{K_{0}}$. Then there exists $\varepsilon>0$ such that $K_{0} \cap B\left(\left(X_{1}^{*}, X_{2}^{*}\right), \varepsilon\right)=\varnothing$, where $B\left(\left(X_{1}^{*}, X_{2}^{*}\right), \varepsilon\right)$ is the ball of center $\left(X_{1}^{*}, X_{2}^{*}\right)$ and radius $\varepsilon>0$. As $\left(X_{1}^{*}, X_{2}^{*}\right)$ is strongly exposed, there exists a linear form $\left(X_{1}, X_{2}\right)$ strongly exposing it, and one can choose $\eta>0$ small enough to ensure $\delta\left[T_{K}\left(\left(X_{1}, X_{2}\right), \eta\right)\right]<\varepsilon$. Since $T_{K}\left(\left(X_{1}, X_{2}\right), \eta\right)$ contains $\left(X_{1}^{*}, X_{2}^{*}\right)$, one concludes that $K_{0} \cap T_{K}\left(\left(X_{1}, X_{2}\right), \eta\right)=\varnothing$, thus

$$
K_{0} \subset\left\{\left(Y_{1}^{*}, Y_{2}^{*}\right) \in K \mid\left\langle X_{1}, Y_{1}^{*}\right\rangle+\left\langle X_{2}, Y_{2}^{*}\right\rangle \geq\left\langle X_{1}, X_{1}^{*}\right\rangle+\left\langle X_{2}, X_{2}^{*}\right\rangle+\eta\right\}
$$

But the right-hand side is a closed convex set, so by taking the closed convex hull of the left-hand side, one gets

$$
\overline{c o}\left(K_{0}\right) \subset\left\{\left(Y_{1}^{*}, Y_{2}^{*}\right) \in K \mid\left\langle X_{1}, Y_{1}^{*}\right\rangle+\left\langle X_{2}, Y_{2}^{*}\right\rangle \geq\left\langle X_{1}, X_{1}^{*}\right\rangle+\left\langle X_{2}, X_{2}^{*}\right\rangle+\eta\right\}
$$

and taking $\left(Y_{1}^{*}, Y_{2}^{*}\right)=\left(X_{1}^{*}, X_{2}^{*}\right) \in K$ leads to a contradiction.

Therefore $\mathcal{E}(K) \subset \overline{K_{0}}$, and one has $\mathcal{E}(C) \times \mathcal{E}(C) \subset \mathcal{E}(K) \subset \overline{K_{0}}$, QED.

By a celebrated theorem of Bishop and Phelps (see again [9]), there is a dense subset $H$ of $L^{2}$ (in fact, a dense $G_{\delta}$ ) such that, for every $X \in H$, the maximum of $\left\langle X^{*}, X\right\rangle$ for $X^{*} \in C$ is attained at a strongly exposed point. Going back to (1.9), take some $X \in H$, and let $X^{*} \in C$ be such that

$$
\varrho(X)=\left\langle X^{*}, X\right\rangle
$$


with $X^{*} \in \mathcal{E}(C)$. Now take another $Y \in H$, and another point $Y^{*} \in \mathcal{E}(C)$ such that $\varrho(Y)=\left\langle Y^{*}, Y\right\rangle$. One has $\left(X^{*}, Y^{*}\right) \in \mathcal{E}(C) \times \mathcal{E}(C)$, and it results from the previous lemma that $\left(X^{*}, Y^{*}\right) \in \overline{K_{0}}$. This implies the existence of a sequence $\left(X_{n}^{*}, Y_{n}^{*}\right) \in K_{0}$ such that $\left(X_{n}^{*}, Y_{n}^{*}\right)$ converges to $\left(X^{*}, Y^{*}\right)$ in norm. By the definition of $K_{0}$, one should have $X_{n}^{*} \sim Y_{n}^{*}$, that is $Y_{n}^{*}=X_{n}^{*} \circ \sigma_{n}$ for $\sigma_{n} \in \mathcal{A}$. Hence, $\varrho(Y)=\left\langle Y^{*}, Y\right\rangle=\lim _{n}\left\langle Y_{n}^{*}, Y\right\rangle=$ $\lim _{n}\left\langle X_{n}^{*} \circ \sigma_{n}, Y\right\rangle=\lim _{n}\left\langle X_{n}^{*}, Y \circ \sigma_{n}^{-1}\right\rangle$. But by the Cauchy-Schwartz inequality,

$$
\left|\left\langle X_{n}^{*}, Y \circ \sigma_{n}^{-1}\right\rangle-\left\langle X^{*}, Y \circ \sigma_{n}^{-1}\right\rangle\right| \leq\left\|Y \circ \sigma_{n}^{-1}\right\|_{2}\left\|X_{n}^{*}-X^{*}\right\|_{2},
$$

which tends to 0 as $\left\|Y \circ \sigma_{n}^{-1}\right\|_{2}=\|Y\|_{2}$. Therefore, $\varrho(Y)=\lim _{n}\left\langle X_{n}^{*}, Y \circ \sigma_{n}^{-1}\right\rangle=$ $\lim _{n}\left\langle X^{*}, Y \circ \sigma_{n}^{-1}\right\rangle \leq \sup _{\tilde{Y} \sim Y}\left\langle X^{*}, \tilde{Y}\right\rangle$. But one has also $\varrho(Y)=\sup _{Y^{*} \in C}\left\langle Y^{*}, Y\right\rangle \geq$ $\sup _{\sigma \in \mathcal{A}}\left\langle X^{*} \circ \sigma, Y\right\rangle=\sup _{\sigma \in \mathcal{A}}\left\langle X^{*}, Y \circ \sigma\right\rangle \geq \sup _{\tilde{Y} \sim Y}\left\langle X^{*}, \tilde{Y}\right\rangle$, therefore

$$
\varrho(Y)=\sup _{\tilde{Y} \sim Y}\left\langle X^{*}, \tilde{Y}\right\rangle \quad \forall Y \in \Omega
$$

The functions $\rho(Y)$ and:

$$
\sup _{\tilde{Y} \sim Y}\left\langle X^{*}, \tilde{Y}\right\rangle=\sup _{\tilde{X} \sim Y}\left\langle\tilde{X}^{*}, Y\right\rangle
$$

are both convex, finite and lsc on $L^{2}$, and hence continuous. Since they coincide on a dense subset, they coincide everywhere. This proves the direct implication (i) $\Rightarrow$ (ii) of the theorem.

We now turn to the converse. Let $\varrho_{\mu}$ be a maximal correlation risk measure with respect to baseline measure $\mu$. Then $\varrho_{\mu}$ is clearly convex. Take $X$ and $Y$ in $L_{d}^{2}$. By proposition 7 in the Appendix, there exist two convex functions $\phi_{1}$ and $\phi_{2}$ such that for $U \sim \mu$, one has $\nabla \phi_{1}(U) \sim X$ and $\nabla \phi_{2}(U) \sim Y$, and $\varrho_{\mu}(X)=E\left[U \cdot \nabla \phi_{1}(U)\right], \varrho_{\mu}(Y)=E\left[U \cdot \nabla \phi_{2}(U)\right]$. Thus $\varrho_{\mu}(X)+\varrho_{\mu}(Y)=E\left[U \cdot\left(\nabla \phi_{1}(U)+\nabla \phi_{2}(U)\right)\right]$, but for all $\tilde{U} \sim U, E\left[U \cdot\left(\nabla \phi_{1}(U)+\nabla \phi_{2}(U)\right)\right] \geq$ $E\left[\tilde{U} \cdot\left(\nabla \phi_{1}(U)+\nabla \phi_{2}(U)\right)\right]$, hence $\varrho_{\mu}(X)+\varrho_{\mu}(Y)=\sup \{\varrho(\tilde{X}+\tilde{Y}): \tilde{X} \sim X, \tilde{Y} \sim Y\}$. Thus $\varrho_{\mu}$ is strongly coherent, which completes the proof of Theorem 1.

\section{A multivariate generalization of Kusuoka's theorem}

In this section we recall the existing axiomatization leading to the representation result of Kusuoka in [15], where risk measures for univariate risks that are subadditive, law invariant and comonotonic additive are represented by maximal correlation functionals. We then propose a way to generalize these axioms to the case where risk measures deal with multivariate 
risks, by showing how to generalize the only problematic axiom, namely comonotonic additivity. We then give a representation result which extends Kusuoka's to the multivariate case.

2.1. Coherent and regular risk measures. To describe the existing axiomatic framework, we first recall the following definitions valid in the univariate case, from [1], and existing results.

Definition 4 (Coherent; Convex risk measures). A functional $\varrho: L^{2} \rightarrow \mathbb{R}$ is called a coherent risk measure if it satisfies the following four properties (MON), (TI), (CO) and (PH) as follows:

- Monotonicity (MON): $X \leq Y \Rightarrow \varrho(X) \leq \varrho(Y)$

- Translation invariance (TI): $\varrho(X+m)=\varrho(X)+m \varrho(1)$

- Convexity (CO): $\varrho(\lambda X+(1-\lambda) Y) \leq \lambda \varrho(X)+(1-\lambda) \varrho(Y)$ for all $\lambda \in[0,1]$.

- Positive homogeneity (PH): $\varrho(\lambda X)=\lambda \varrho(X)$ for all $\lambda \geq 0$.

A functional which only satisfies (MON), (TI) and (CO) is called a convex risk measure.

Even though these definitions are mostly standard, note that since we have considered risk measures associated with random vectors of potential losses, the definition of monotonicity takes an non decreasing form, unlike the definition in most of the literature on coherent risk measures. Compared to the traditional presentation in the literature, the expression of translation invariance is adapted to take into account the fact that we did not impose the scaling convention $\varrho(1)=1$. Also note (as we have a multivariate generalization in mind) that, let alone monotonicity (which we shall discuss separately below), all these axioms admit a straightforward generalization to the case of risks $X \in L_{d}^{2}$. The expression for $(\mathrm{CO})$ and $(\mathrm{PH})$ will remain unchanged; for (TI) the natural extension to dimension $d$ will be given in (2.2) below.

A representation of coherent risk measures was given in the original work of [1], whereas representation of convex risk measures was proposed in [10]. These were extended to the multivariate setting by Jouini, Meddeb and Touzi in [13] who characterize coherent acceptance sets, i.e. sets in $\mathbb{R}^{n}$ that cancel the risk associated with an $\mathbb{R}^{d}$ valued random vector, 
and consider aggregation issues, and Burgert and Rüschendorf in [4] who characterize convex real valued measures for multivariate risks, and Rüschendorf in [17], who characterizes those of the latter that are law invariant, and proposes maximal correlation risk measures as an example. The idea of introducing a variational characterization of comonotonic additivity as well as the generalization of Kusuoka's axiomatic approach it allows constitute the essential novelties of this section.

Regularity. In the case of univariate risks, comonotonic additivity is used in addition to law invariance to define regular risk measures (see [10], sect. 4.7):

Definition 5 (Comonotonicity; Regularity). Two random variables $X$ and $Y$ are comonotonic (or synonymously, comonotone) if there exits a random variable $U$ and two increasing functions $\phi$ and $\psi$ such that $X=\phi(U)$ and $Y=\psi(U)$ hold almost surely.

A functional $\varrho: L^{2} \rightarrow \mathbb{R}$ is called a regular risk measure if it satisfies:

- Law invariance (LI), and

- Comonotonic additivity $(C A): \varrho(X+Y)=\varrho(X)+\varrho(Y)$ when $X, Y$ are comonotonic.

Note that comonotonic additivity implies translation invariance, as any random variable is comonotonic with the constant. Informally speaking, law invariance suggests that the risk measure is a functional of the quantile function $F_{X}^{-1}(t)=\inf \left\{x: F_{X}(x) \geq t\right\}$ associated with the distribution. Positive homogeneity and comonotonic additivity together suggest that this representation is linear $\varrho(X):=\int_{0}^{1} \phi(t) F_{X}^{-1}(t) d t$. Finally, subadditivity suggests that the weights $\phi(t)$ are increasing with respect to $t$. Precisely Kusuoka has shown the following in [15], Theorem 7:

Proposition 2 (Kusuoka). A coherent risk measure $\varrho$ is regular if and only if for some increasing and nonnegative function $\phi$ on $[0,1]$, we have

$$
\varrho(X):=\int_{0}^{1} \phi(t) F_{X}^{-1}(t) d t
$$

where $F_{X}$ denotes the cumulative distribution functions of the random variable $X$, and its generalized inverse $F_{X}^{-1}(t)=\inf \left\{x: F_{X}(x) \geq t\right\}$ is the associated quantile function. 
Variational characterization. By the Hardy-Littlewood-Pólya inequality shown in lemma 11 of [15], we can write a variational expression for coherent regular risk measures:

$$
\int_{0}^{1} \phi(t) F_{X}^{-1}(t) d t=\max \{\mathbb{E}[X \tilde{U}]: \tilde{U} \sim \mu\}
$$

where $\mu$ if the probability distribution of $\phi$, and the maximum is taken over the equidistribution class of $\mu$. The reader is referred to [7] and [6] for a nice treatment of this variational problem and the dual representation of Schur convex functions in the univariate case. As we shall see, variational characterization 2.1 will be key when generalizing to the multivariate setting.

2.2. A multivariate notion of comonotonicity. We now turn to an extension of the concept of comonotonicity. Note first that a valid definition of comonotonicity in dimension one is the following: two random variables $X$ and $Y$ are comonotonic if and only if one can construct almost surely $Y=T_{Y}(U)$ and $X=T_{X}(U)$ for some third random variable $U$, and $T_{X}, T_{Y}$ non decreasing functions. In other words, $X$ and $Y$ are comonotonic whenever there is a random variable $U$ such that $\mathbb{E}[U X]=\max \{\mathbb{E}[X \tilde{U}]: \tilde{U} \sim U\}$ and $\mathbb{E}[U Y]=\max \{\mathbb{E}[Y \tilde{U}]: \tilde{U} \sim U\}$. This variational characterization will be the basis for our generalized notion of comonotonicity.

To simplify our exposition in the remainder of the paper, we shall make the following assumption:

Assumption. In the remainder of the paper, we shall assume that the baseline distribution of risk $\mu$ is absolutely continuous with respect to Lebesgue measure.

Definition 6 ( $\mu$-comonotonicity). Let $\mu$ be a probability measure on $\mathbb{R}^{d}$ that is absolutely continuous. Two random vectors $X$ and $Y$ in $L_{d}^{2}$ are called $\mu$-comonotonic if for some random vector $U \sim \mu$, we have

$$
\begin{aligned}
& U \in \operatorname{argmax}_{\tilde{U}}\{\mathbb{E}[X \cdot \tilde{U}], \tilde{U} \sim \mu\}, \text { and } \\
& U \in \operatorname{argmax}_{\tilde{U}}\{\mathbb{E}[Y \cdot \tilde{U}], \tilde{U} \sim \mu\} .
\end{aligned}
$$


In particular, every random vector $X$ is $\mu$-comonotonic with constant vectors $Y=y$. Note that the geometric interpretation of this definition is that $X$ and $Y$ are $\mu$-comonotonic if and only if they have the same $L^{2}$ projection on the equidistribution class of $\mu$. We next give a few useful lemmas. We start with a result securing the existence of a $\mu$-comonotonic pair with given marginals.

Lemma 7. Let $\mu$ be a probability measure on $\mathbb{R}^{d}$ that is absolutely continuous. Then given two probability distributions $P$ and $Q$ in $\mathcal{P}_{2}\left(\mathbb{R}^{d}\right)$, there exists a pair $(X, Y)$ in $\left(L_{d}^{2}\right)^{2}$ such that $X \sim P, Y \sim Q$, and $X$ and $Y$ are $\mu$-comonotonic.

Proof. By Brenier's theorem (Proposition 7 in the Appendix), there exists $U \sim \mu$ and two convex functions $\phi_{1}$ and $\phi_{2}$ such that $X=\nabla \phi_{1}(U) \sim P$ and $Y=\nabla \phi_{2}(U) \sim Q$. Then $X$ and $Y$ are $\mu$-comonotonic.

We then provide a useful characterization of $\mu$-comonotonicity.

Lemma 8. Let $\mu$ be probability measure on $\mathbb{R}^{d}$ that is absolutely continuous. Then two random vectors $X$ and $Y$ in $L_{d}^{2}$ are $\mu$-comonotonic if

$$
\varrho_{\mu}(X+Y)=\varrho_{\mu}(X)+\varrho_{\mu}(Y)
$$

where $\varrho_{\mu}(X):=\sup \{\mathbb{E}[X \cdot \tilde{U}]: \tilde{U} \sim \mu\}$ is the maximal correlation risk measure, defined in Definition 3 above.

Proof. There exists $U \sim \mu$ such that $\varrho_{\mu}(X+Y)=\mathbb{E}[(X+Y) \cdot U]$. We have $\mathbb{E}[(X+Y) \cdot U]=$ $\mathbb{E}[X \cdot U]+\mathbb{E}[Y \cdot U]$, and both inequalities $\mathbb{E}[X \cdot U] \leq \varrho_{\mu}(X)$ and $\mathbb{E}[Y \cdot U] \leq \varrho_{\mu}(Y)$ hold, thus $\mathbb{E}[X \cdot U]+\mathbb{E}[Y \cdot U] \leq \varrho_{\mu}(X)+\varrho_{\mu}(Y)$ with equality if and only both inequalities above are actually equalities, which is the equivalence needed.

This lemma implies in particular that maximal correlation functionals with baseline measure $\mu$ are $\mu$-comonotone additive. Thus combining with Theorem 1 , this establishes that strongly coherent risk measures are $\mu$-comonotone additive for some $\mu$.

We next show that in dimension 1 , the notion of $\mu$-comonotonicity is equivalent to the classical notion of comonotonicity, regardless of the choice of $\mu$ (provided it is absolutely continuous). 
Lemma 9. In dimension $d=1$, let $\mu$ be probability measure on $\mathbb{R}^{d}$ that is absolutely continuous. Then $X$ and $Y$ are $\mu$-comonotonic if and only if they are comonotonic in the classical sense, that is, if and only if there exists a random variable $Z$ and two non decreasing functions $f$ and $g$ such that $X=f(Z)$ and $Y=g(Z)$ holds almost surely.

Proof. Suppose that $X$ and $Y$ are $\mu$-comonotonic. Then there is a $U \sim \mu$ such that $U \in$ $\operatorname{argmax}_{\tilde{U}}\{\mathbb{E}[X \tilde{U}], \tilde{U} \sim \mu\}$ and $U \in \operatorname{argmax}_{\tilde{U}}\{\mathbb{E}[Y \tilde{U}], \tilde{U} \sim \mu\}$. This implies in particular the existence of two increasing functions $f$ and $g$ such that $X=f(U)$ and $Y=g(U)$ holds almost surely. Hence $X$ and $Y$ are comonotonic in the classical sense. Conversely, suppose that $X$ and $Y$ are comonotonic in the classical sense. There exists a random variable $Z$ and two increasing functions $f$ and $g$ such that $X=f(Z)$ and $Y=g(Z)$ holds almost surely. Let $F_{Z}$ be the cumulative distribution function of $Z$, and $F_{\mu}$ the one associated with $\mu$. Defining $U=F_{\mu}^{-1}\left(F_{Z}(Z)\right)$, one has $U \sim \mu$, and denoting $\varphi=f \circ F_{\mu}^{-1} \circ F_{Z}$ and $\phi=g \circ F_{\mu}^{-1} \circ F_{Z}$, one has $X=\varphi(U)$ and $Y=\phi(U)$. Thus $X$ and $Y$ are $\mu$-comonotonic.

In dimension one, one recovers the classical notion of comonotonicity regardless of the choice of $\mu$ as shown in the previous lemma. However, in dimension greater than one, the comonotonicity relation crucially depends on the baseline distribution $\mu$, unlike in dimension one. The following lemma makes this precise.

Lemma 10. Let $\mu$ and $\nu$ be probability measures on $\mathbb{R}^{d}$ that is absolutely continuous. Then:

- In dimension $d=1$, $\mu$-comonotonicity always implies $\nu$-comonotonicity.

- In dimension $d \geq 2, \mu$-comonotonicity implies $\nu$-comonotonicity if and only if $\nu=T \# \mu$ for some location-scale transform $T(u)=\lambda u+u_{0}$ where $\lambda>0$ and $u_{0} \in \mathbb{R}^{d}$. In other words, comonotonicity is an invariant of the location-scale family transformation classes.

Proof. In dimension one, all the notions of $\mu$-comonotonicity coincide with the classical notion of comonotonicity, as remarked above. Let $d \geq 2$, and suppose that $\mu$-comonotonicity implies $\nu$-comonotonicity. Consider $U \sim \mu$, and let $\phi$ be the convex function (defined up to an additive constant) such that $\nabla \phi \# \nu=\mu$. Then there exists a random vector $V \sim \nu$ such that $U=\nabla \phi(V)$ almost surely. Consider some arbitrary symmetric positive endomorphism $\Sigma$ acting on $\mathbb{R}^{d}$. Then the map $u \rightarrow \Sigma(u)$ is the gradient of a convex function (namely the 
associated quadratic form $\left.u \rightarrow \frac{1}{2}\langle u, \Sigma(u)\rangle\right)$, therefore the random vectors $U$ and $\Sigma(U)$ are $\mu$-comonotonic. By hypothesis, it follows that $U$ and $\Sigma(U)$ are also $\nu$-comonotonic, hence there exists a convex function $\zeta$ such that $\Sigma(U)=\nabla \zeta(V)$ holds almost surely. Therefore, the equality $\Sigma \circ \nabla \phi(v)=\nabla \zeta(v)$ holds for almost every $v$. By differentiating twice (which can be done almost everywhere, by Aleksandrov's theorem), we get that $\Sigma \circ D^{2} \phi(v)=$ $D^{2} \zeta(v)$ hence $\Sigma \circ D^{2} \phi$ is almost everywhere a symmetric endomorphism. This being true regardless of the choice of $\Sigma$, it follows that the matrix of $D^{2} \phi$ in any orthonormal basis of $\mathbb{R}^{d}$ is almost everywhere a diagonal matrix, hence there exists a real valued map $\lambda(u)$ such that $D^{2} \phi(u)=\lambda(u) u$, with $\lambda(u)>0$. But this implies $\partial_{u_{i}} \partial_{u_{j}} \phi(u)=0$ for $i \neq j$ and $\partial_{u_{i}}^{2} \phi(u)=\lambda(u)$ for all $i$. Therefore, $\partial_{u_{j}} \lambda(u)=\partial_{u_{j}} \partial_{u_{i}}^{2} \phi(u)=0$. Hence $\lambda(u)=\lambda$ a strictly positive constant. It follows that $\nabla \phi(u)=\lambda u+u_{0}$, QED. The converse holds trivially.

Remark 2. A close inspection of the proof of this lemma reveals that the essential reason of the discrepancy between dimension one and higher is the simple fact that the general linear matrix group $\mathcal{G} l_{d}(\mathbb{R})$ is Abelian if and only if $d=1$.

We can now define a concept which generalizes comonotonic additivity to the multidimensional setting.

Definition 7 ( $\mu$-comonotonic additivity; $\mu$-regularity). A functional $\varrho: L_{d}^{2} \rightarrow \mathbb{R}$ is called a $\mu$-regular risk measure if it satisfies:

- Law invariance (LI), and

- $\mu$-comonotonic additivity $(\mu-C A): \varrho(X+Y)=\varrho(X)+\varrho(Y)$ when $X, Y$ are $\mu$ comonotonic.

As every random vector is comonotonic with constants, it implies that a $\mu$-comonotonic additive functional $\varrho$ is in particular translation invariant in the following multivariate sense

$$
\varrho(X+m y)=\varrho(X)+m \varrho(y) \text { for all } m \in \mathbb{R} \text { and } y \in \mathbb{R}^{d} .
$$

2.3. A multivariate extension of Kusuoka's theorem. We now show that maximal correlation is equivalent to the combination of subadditivity, law invariance, $\mu$-comonotonic 
additivity and positive homogeneity. Further, the probability measure $\mu$ involved in the definition of comonotonic additivity shall be precisely related to the one which is taken as a baseline scenario of the maximal correlation measure.

We have seen above (lemma 8) that maximal correlation risk measures defined with respect to a distribution $\mu$ are $\mu$-comonotonic additive. When the measure is also law invariant and coherent, we shall see that the converse holds true, and this constitutes our second main result, which is a multivariate extension of Kusuoka's theorem. Note that while Kusuoka's theorem was stated using the axioms of subadditivity and positive homogeneity in addition to others, we only need the weaker axiom of convexity in addition to the same others.

Theorem 2. Let $\varrho$ be a l.s.c. risk measure on $L_{d}^{2}$ with the properties of convexity (CO), and $\mu$-regularity, that is law invariance (LI) and $\mu$-comonotonic additivity ( $\mu$-CA). Then $\varrho$ is strongly coherent. Equivalently, @ is a maximal correlation risk measure, namely there exists $\nu \in \mathcal{P}_{2}\left(\mathbb{R}^{d}\right)$ such that $\varrho=\varrho_{\nu}$, where $\varrho_{\nu}$ is a maximal correlation measure with respect to baseline scenario $\nu$, and $\mu$ and $\nu$ are related by location-scale transformation, that is $\nu=T \# \mu$ where $T(u)=\lambda u+u_{0}$ with $\lambda>0$ and $u_{0} \in \mathbb{R}^{d}$.

Proof. Combining the convexity and law invariance axioms imply $\varrho(\tilde{X}+\tilde{Y}) \leq \varrho(X)+\varrho(Y)$ for all $X, Y, \tilde{X}, \tilde{Y}$ in $L_{d}^{2}$, thus $\varrho(X)+\varrho(Y) \geq \sup \{\varrho(\tilde{X}+\tilde{Y}): \tilde{X} \sim X ; \tilde{Y} \sim Y\}$. But by Lemma 7 , there exists a $\mu$-comonotonic pair $(X, Y)$. By $\mu$-comonotonic additivity, one has $\varrho(X)+\varrho(Y)=\varrho(X+Y)$, therefore the previous inequality is actually an equality, and

$$
\varrho(X)+\varrho(Y)=\sup \{\varrho(\tilde{X}+\tilde{Y}): \tilde{X} \sim X ; \tilde{Y} \sim Y\}
$$

therefore $\varrho$ is strongly coherent. By Theorem 1 , it results that there exists $\nu \in \mathcal{P}_{2}\left(\mathbb{R}^{d}\right)$ such that $\varrho=\varrho_{\nu}$. But by the comonotonic additivity of $\varrho$ and lemma 8 , any two vectors $X$ and $Y$ which are $\mu$-comonotonic are also $\nu$-comonotonic. By lemma 10, this implies that there is a location-scale map $T$ such that $\nu=T \# \mu$, so that the result follows.

Because it allows a natural generalization of well-known univariate results, this theorem makes a strong point in arguing that our notion of comonotonic additivity is the right one when considering multivariate risks. 
2.4. Extending monotonicity. We extend the concept of monotonicity with reference to a partial order $\preceq$ defined on $\mathbb{R}^{d}$ in the following way:

Definition 8 ( $\preceq$-monotonicity). A functional $\varrho: L^{2} \rightarrow \mathbb{R}$ is said to be $\preceq$-monotone if it satisfies:

$(\preceq-M O N): X \preceq Y$ almost surely $\Rightarrow \varrho(X) \leq \varrho(Y)$.

We have the following result:

Proposition 3. Let $\varrho_{\mu}$ be the maximal correlation risk measure with respect to baseline distribution $\mu$. Let $(\text { Supp } \mu)^{0}$ be the polar cone of the support of $\mu$. For a cone $C \subset \mathbb{R}^{d}$, denote $\preceq_{C}$ the partial order in $\mathbb{R}^{d}$ induced by $C$, namely $x \preceq_{C} y$ if and only if $y-x \in C$. Then $\varrho_{\mu}$ is monotone with respect to $\preceq_{C}$ if and only if $C \subset-(\operatorname{Supp} \mu)^{0}$.

Proof. If $X$ and $U$ are $\mu$-comonotonic, then $D \varrho_{X}(Z)=E[U \cdot Z]$, but the property that $E[U \cdot Z] \geq 0$ for all $Z$ almost surely included in $C$ is equivalent to $C \subset-(\operatorname{Supp} \mu)^{0}$.

Note that in dimension $d=1$, with $C=\mathbb{R}_{+}$, one recovers the usual notion of monotonicity. In higher dimension, we get in particular that if $\mu$ is supported in $\mathbb{R}_{+}^{d}$, then $\varrho_{\mu}$ is monotone with respect to the strong order of $\mathbb{R}^{d}$. Finally, note also that the concept of monotonicity proposed here is a somewhat weak one, as it deals only with almost sure domination between $X$ and $Y$. A stronger concept of monotonicity would involve stochastic ordering of $X$ and $Y$; we do not pursue this approach here.

\section{NumERicAl COMPUTATION}

In this section, we show explicit examples of computation of the maximal correlation risk measure. We start by the Gaussian case, where closed-form formulas are available. To handle more general cases we shall show that the problem may be thought of as an auction mechanism, an intuition we shall develop and use to derive an efficient computational algorithm. 
3.1. Gaussian risks. We now consider the case where the baseline risk $U$ is Gaussian with distribution $\mu=N\left(0, \Sigma_{U}\right)$, with $\Sigma_{U}$ a positive definite matrix of size $d$, and we study the restriction of $\varrho_{\mu}$ to the class of Gaussian risks.

Note (cf. [16] I, Ex. 3.2.12) that the linear map $u \rightarrow A_{X} u$ where

$$
A_{X}=\Sigma_{U}^{-1 / 2}\left(\Sigma_{U}^{1 / 2} \Sigma_{X} \Sigma_{U}^{1 / 2}\right)^{1 / 2} \Sigma_{U}^{-1 / 2}
$$

sends the probability measure $N\left(0, \Sigma_{U}\right)$ to the probability measure $N\left(0, \Sigma_{X}\right)$; further, $A_{X}$ is positive semidefinite, so this map is the gradient of convex function $u \rightarrow \frac{1}{2} u^{\prime} A_{X} u$. Hence we have the following straightforward matrix formulation of comonotonicity.

Lemma 11. Consider two Gaussian vectors $X \sim N\left(0, \Sigma_{X}\right)$ and $Y \sim N\left(0, \Sigma_{Y}\right)$ with $\Sigma_{X}$ and $\Sigma_{Y}$ invertible. Then $X$ and $Y$ are $\mu$-comonotonic if and only if

$$
E\left[X Y^{T}\right]=\Sigma_{U}^{-1 / 2}\left(\Sigma_{U}^{1 / 2} \Sigma_{X} \Sigma_{U}^{1 / 2}\right)^{1 / 2}\left(\Sigma_{U}^{1 / 2} \Sigma_{Y} \Sigma_{U}^{1 / 2}\right)^{1 / 2} \Sigma_{U}^{-1 / 2}
$$

In particular, in the case $\mu=N\left(0, I_{d}\right), X$ and $Y$ are $\mu$-comonotonic if and only if $E\left[X Y^{T}\right]=$ $\Sigma_{X}^{1 / 2} \Sigma_{Y}^{1 / 2}$

Proof. If $X$ and $Y$ are $\mu$-comonotonic, then there exists $U \sim N\left(0, \Sigma_{U}\right)$ such that $X=A_{X} U$ and $Y=A_{Y} U$, and the result follows. Conversely, if equality (3.1) holds, then denoting $U=A_{X}^{-1} X$ and $V=A_{Y}^{-1} Y$, we get that 1) $U \sim N\left(0, \Sigma_{U}\right)$ and $V \sim N\left(0, \Sigma_{U}\right)$, and 2) $E\left[U V^{T}\right]=A_{X}^{-1} E\left[X Y^{T}\right] A_{Y}^{-1}=\Sigma_{U}$, therefore by the Cauchy-Schwartz inequality, $U=V$ almost surely. Thus $X$ and $Y$ are $\mu$-comonotonic.

We now derive the value of correlation risk measures at Gaussian risks. Still by [16] I, Ex. 3.2.12, we have immediately:

Proposition 4. When the baseline risk $U$ is Gaussian with distribution $\mu=N\left(0, \Sigma_{U}\right)$, we have for a Gaussian vector $X \sim N\left(0, \Sigma_{X}\right)$ :

$$
\varrho_{\mu}(X)=\operatorname{tr}\left[\left(\Sigma_{U}^{1 / 2} \Sigma_{X} \Sigma_{U}^{1 / 2}\right)^{1 / 2}\right] .
$$

In particular, in the case $\mu=N\left(0, I_{d}\right), \varrho_{\mu}$ is the trace norm: $\varrho_{\mu}(X)=\operatorname{tr}\left[\Sigma_{X}^{1 / 2}\right]$. 
Proof. One has $\varrho_{\mu}(X)=\max \{\mathbb{E}[\tilde{X} \cdot U] ; \tilde{X} \sim X\}=\mathbb{E}\left[A_{X} U U^{T}\right]$, thus because of the previous results, $\varrho_{\mu}(X)=\mathbb{E}\left[U^{T} \Sigma_{U}^{-1 / 2}\left(\Sigma_{U}^{1 / 2} \Sigma_{X} \Sigma_{U}^{1 / 2}\right)^{1 / 2} \Sigma_{U}^{-1 / 2} U\right]=\operatorname{tr}\left(\left(\Sigma_{U}^{1 / 2} \Sigma_{X} \Sigma_{U}^{1 / 2}\right)^{1 / 2}\right)$.

In dimension 2 , we have the formula $\operatorname{tr}(\sqrt{S})=\sqrt{\operatorname{tr}(S)+2 \sqrt{\operatorname{det} S}}$, so we get a closed form expression:

Example 3. When $d=2$, and $\mu=N\left(0, I_{2}\right)$, we have for $\Sigma_{X}=\left(\begin{array}{cc}\sigma_{1}^{2} & \varrho \sigma_{1} \sigma_{2} \\ \varrho \sigma_{1} \sigma_{2} & \sigma_{2}^{2}\end{array}\right)$ the following expression $\varrho_{\mu}(X)=\sqrt{\sigma_{1}^{2}+\sigma_{2}^{2}+2 \sigma_{1} \sigma_{2} \sqrt{1-\varrho^{2}}}$.

3.2. Kantorovich duality and Walras auction. We now see how optimal transportation duality permits the computation of maximal correlation risk measures. More precisely, we shall see that the algorithm we shall propose to compute numerically the maximal correlation risk measures is to be thought of intuitively as a Walrasian auction, as we shall explain. We refer to [16] and [22] for overviews of the theory and applications of optimal transportation, including recent results. Consider a baseline distribution $\mu$, and recall the expression for the maximal correlation risk measure $\varrho_{\mu}(X)$ of a random vector $X \in \mathbb{R}^{d}: \varrho(X)=\sup \{\mathbb{E}[X \cdot \tilde{U}]: \tilde{U} \sim \mu\}$. This problem is the problem of computing the maximal transportation cost of mass distribution $\mu$ to mass distribution $\mathcal{L}_{X}$ with cost of transportation $c(u, x)=u \cdot x$.

The problem has a dual expression according to Monge-Kantorovich duality (or duality of optimal transportation). We have (theorem 2.9 page 60 of [22]):

$$
\varrho_{\mu}(X)=\min _{V \in \text { c.l.s.c. }\left(\mathbb{R}^{d}\right)}\left(\int V d \mu+\int V^{*} d \mathcal{L}_{X}\right) .
$$

The function $V$ that achieves the minimum in (3.2) exists by theorem 1(iii) and when $\mathcal{L}_{X}$ is absolutely continuous, one has $\nabla V^{*}(X) \sim \mu$ and $\varrho_{\mu}(X)=\mathbb{E}\left[X \cdot \nabla V^{*}(X)\right]$. In the sequel we shall make the law invariance of $\varrho_{\mu}$ and the symmetry between the roles played by the distributions of $X$ and $U$ explicit in the notation by writing

$$
\varrho_{\mu}\left(\mathcal{L}_{X}\right):=\varrho\left(\mu, \mathcal{L}_{X}\right):=\varrho_{\mu}(X)
$$


3.2.1. Law-invariant, convex risk measures. Following [17], theorem 2.3, the maximum correlation risk measures are the building blocks of more general convex risk measures. One has the following result, which was proven by Rüschendorf in the cited paper.

Proposition 5. Let $\varrho$ be a convex measure. Then $\varrho$ is law invariant if and only if there exists a penalty function $\alpha$ such that

$$
\varrho(X)=\sup _{\mu \in \mathcal{P}_{2}\left(\mathbb{R}^{d}\right)} \varrho_{\mu}(X)-\alpha(\mu)
$$

Furthermore, $\alpha(\mu)$ can be chosen as $\alpha(\mu)=\sup \left\{\varrho_{\mu}(X): X \in L_{d}^{2}, \varrho(X) \leq 0\right\}$.

3.2.2. Dual representations of the risk measure. The following lemma provides an expression of the conjugate of the maximal correlation risk measure.

Lemma 12. For $W: \mathbb{R}^{d} \rightarrow \mathbb{R}$ convex and lower semicontinuous, one has

$$
\sup _{P \in \mathcal{P}_{2}\left(\mathbb{R}^{d}\right)}\left\{\varrho_{\mu}(P)+\int W d P\right\}=\int(-W)^{*} d \mu .
$$

Proof. One has $\int(-W)^{*} d \mu=\int \sup _{y}\{u \cdot y+W(y)\} d \mu(u)$, thus $\int(-W)^{*} d \mu=\sup _{\tau(\cdot)} \int u$. $\tau(u)+W(\tau(u)) d \mu(u)$ where the supremum is over all measurable maps $\tau: \mathbb{R} \rightarrow \mathbb{R}$. Grouping by equidistribution class, one has

$$
\begin{aligned}
\int(-W)^{*} d \mu & =\sup _{P}\left[\sup _{\tau \# \mu=P} \int u \cdot \tau(u) d \mu(u)+\int W d P\right] \\
& =\sup _{P}\left\{\varrho_{\mu}(P)+\int W d P\right\} .
\end{aligned}
$$

3.2.3. General equilibrium interpretation. We now consider then $\varrho\left(\mu, \mathcal{L}_{X}\right)$ for two probability distributions on $\mathbb{R}^{d}$, and we interpret $\mu$ as a distribution of consumers (e.g. insurees) and $\mathcal{L}_{X}$ as a distribution of goods (e.g. insurance contracts) in an economy. Consumer with characteristics $u$ derives utility from the consumption of good with attributes $x$ equal to the interaction $u \cdot x$ of consumer characteristics and good attributes. Consumer $u$ maximizes utility $u \cdot x$ of consuming good $x$ minus the price $V^{*}(x)$ of the good. Hence his 
indirect utility is $\sup _{x \in \mathbb{R}^{d}}\left[u \cdot x-V^{*}(x)\right]=V^{* *}(u)=V(u)$. According to equation (3.2), the total surplus in the economy $\mathbb{E}[X \cdot U]$ is maximized for the pair $\left(V, V^{*}\right)$ of convex lower semi-continuous functions on $\mathbb{R}^{d}$ that minimizes

$$
\Phi(V):=\int V d \mu+\int V^{*} d \mathcal{L}_{X}
$$

The functional $\Phi$ is convex and its Fréchet derivative, when it exists, is interpreted as the excess supply in the economy, with value at $h$ equal to $D \Phi(h)=\int h d\left(\mu-\nu_{V}\right)$, where $\nu_{V}:=\nabla V^{*} \# \mathcal{L}_{X}$. Indeed, the convexity of the map $V \rightarrow \Phi(V)$ follows from the identity established above in lemma 12

$$
\Phi(V)=\sup _{\nu \in \mathcal{P}_{2}(\mathbb{R})^{d}}\left\{\varrho\left(\mathcal{L}_{X}, \nu\right)+\int V d(\mu-\nu)\right\},
$$

thus this map is the supremum of functionals that are linear in $V$. The supremum is attained for $\nu=\nu_{V}$, hence it follows that $D \Phi_{V}(h)=\int h d\left(\mu-\nu_{V}\right)$.

Hence, excess supply is zero when the indirect utility $V$ and the prices $V^{*}$ are such that $\nu_{V}=\mu$. With our economic interpretation above, this can be seen as a Walrasian welfare theorem, where the total surplus is maximized by the set of prices that equates excess supply to zero.

This general equilibrium interpretation of maximal correlation risk measures extends to the method of computation of the latter through a gradient algorithm to minimize the convex functional $\Phi$. This algorithm can be interpreted as a Walrasian tâtonnement that adjusts prices to reduce excess supply $D \Phi_{V}$. This algorithm is described in more detail and implemented fully in the case of discretely distributed risks below.

3.3. Discrete risks. We now consider the restriction $\varrho_{\mu}$ to the class of risks whose distribution is discrete. We have in mind in particular the empirical distribution of a sample of recorded data of the realization of the risk. The procedure we shall now describe consists in the computation of the generalized quantile of the discrete distribution, which opens the way for econometric analysis of maximal correlation risk measures.

3.3.1. Representation. Let $X \sim P_{n}$, where $P_{n}=\sum_{k=1}^{n} \pi_{k} \delta_{Y_{k}}$ is a discrete distribution supported by $\left\{Y_{1}, \ldots, Y_{n}\right\}, n$ distinct points in $\mathbb{R}^{d}$. For instance if $P_{n}$ is the empirical 
measure of the sample $\left\{Y_{1}, \ldots, Y_{n}\right\}$, then $\pi_{k}=1 / n$. We are looking for $\varphi:[0,1]^{d} \rightarrow \mathbb{R}^{d}$ such that:

(i) for (almost) all $u \in[0,1]^{d}, \varphi(u) \in\left\{Y_{1}, \ldots, Y_{n}\right\}$

(ii) for all $k \in\{1, \ldots, n\}, \mu\left(\varphi^{-1}\left\{Y_{k}\right\}\right)=\pi_{k}$ ie. $\varphi$ pushes forward $\mu$ to $P_{n}$

(iii) $\varphi=\nabla V$, where $V: \mathbb{R}^{d} \rightarrow \mathbb{R}$ is a convex function.

It follows from the Monge-Kantorovich duality that there exist weights $\left(w_{1}, \ldots, w_{n}\right) \in \mathbb{R}^{n}$, such that $V(u)=w^{*}(u):=\max _{k}\left\{\left\langle u, Y_{k}\right\rangle-w_{k}\right\}$ is the solution. Introduce the functional $\Phi_{\mu}: \mathbb{R}^{n} \rightarrow \mathbb{R}, \Phi_{\mu}(w)=\int w^{*}(u) d \mu(u)$. The numerical implementation of the method is based on the following result:

Proposition 6. There exist unique (up to an additive constant) weights $w_{1}, \ldots, w_{n}$ such that for $w^{*}(u)=\max _{k}\left\{\left\langle u, Y_{k}\right\rangle-w_{k}\right\}$, the gradient map $\varphi=\nabla w^{*}$ satisfies (i), (ii) and (iii) above. The function $w \rightarrow \Phi_{\mu}(w)+\sum_{k=1}^{n} \pi_{k} w_{k}$ is convex, and reaches its minimum at $w=\left(w_{1}, \ldots, w_{n}\right)$ defined above.

Proof. By the Knott-Smith optimality criterion (theorem 2.12(i) page 66 of [22]), there exists a convex function $w$ on the set $\left\{Y_{1}, \ldots, Y_{n}\right\}$ such that the optimal pair in (3.2) is $(w, V)$, where $V$ is the Legendre-Fenchel conjugate of $w$, i.e. the function $V(u)=$ $\sup _{x \in\left\{Y_{1}, \ldots, Y_{n}\right\}}(u \cdot x-w(x))=\max _{k}\left(u \cdot Y_{k}-w_{k}\right)$, where $w_{k}=w\left(Y_{k}\right)$ for each $k=1, \ldots, n$. Note that the subdifferential $\partial V$ is a singleton except at the boundaries of the sets $\mathcal{U}_{k}=$ $\left\{u: \arg \max _{i}\left\{\left\langle u, Y_{i}\right\rangle-w_{i}\right\}=k\right\}$, so $\nabla V$ is defined $\mathcal{L}_{U}$ almost everywhere. Since for all $k$, and all $u \in \mathcal{U}_{k}, Y_{k} \in \partial V(u), \nabla V$ satisfies (i). Finally, by Brenier's Theorem (theorem 2.12 (ii) page 66 of [22]), $\nabla V$ pushes $\mathcal{L}_{U}$ forward to $P_{n}$, hence it also satisfies (iii). The function $\Phi_{\mu}: w \rightarrow \int w^{*}(u) d \mu(u)$ is convex, which follows from the equality

$$
\int w^{*}(u) d \mu(u)=\max _{\sigma(.)} \int\left\langle u, Y_{\sigma(u)}\right\rangle-w_{\sigma(u)} d \mu(u)
$$

where the maximum is taken over all measurable functions $\sigma: \mathbb{R}^{d} \rightarrow\{1, \ldots, n\}$.

3.3.2. The Tâtonnement Algorithm. The problem is therefore to minimize the convex function $w \rightarrow \Phi_{\mu, \pi}(w)=\Phi_{\mu}(w)+\sum_{k=1}^{n} \pi_{k} w_{k}$, which can be done using a gradient approach. 
To the best of our knowledge, the idea of using the Monge-Kantorovich duality to compute the weights using a gradient algorithm should be credited to F. Aurenhammer and his coauthors. See [2] and also [19]. However, by the economic interpretation seen above, the algorithm's dynamics is the time-discretization of a "tâtonnement process," as first imagined by Léon Walras (1874) and formalized by Paul Samuelson (1947) (see [20]). Hence to emphasize the economic interpretation, we shall refer to the algorithm as "Tâtonnement Algorithm".

The Algorithm. Initialize the prices $w^{0}=0$. At each step $m$, compute $\Phi_{\mu, \pi}\left(w^{m}\right)$ and the excess demand $\nabla \Phi_{\mu, \pi}\left(w^{m}\right)$. For a well chosen elasticity parameter $\epsilon^{m}$, update the prices proportionally to excess demand

$$
w^{m+1}=w^{m}+\epsilon^{m} \nabla \Phi_{\mu, \pi}\left(w^{m}\right)
$$

Go to next step, or terminate the algorithm when the excess demand becomes smaller than a prescribed level.

This algorithm requires the evaluation of the function and its gradient. For this we shall need to compute in turns, for each $\left.k: 1) \mathcal{U}_{k}=\left\{u: \arg \max _{i}\left\{\left\langle u, Y_{i}\right\rangle-w_{i}\right\}=k\right\} ; 2\right)$ $p_{k}=\mu\left(\mathcal{U}_{k}\right)$; and 3) $u_{k}$ the barycenter of $\left(\mathcal{U}_{k}, \mu\right)$ (that is $u_{k}=\mu\left(\mathcal{U}_{k}\right)^{-1} \int_{\mathcal{U}_{k}} z d \mu(z)$.) Then we get the value of $\Phi_{\mu, \pi}(w): \Phi_{\mu, \pi}(w)=\sum\left(\left\langle u_{k}, Y_{k}\right\rangle-w_{k}\right) p_{k}+w_{k} \pi_{k}$ and the value of its gradient $\nabla \Phi_{\mu, \pi}(w)=\pi-p$, ie. $\frac{\partial \Phi_{\mu, \pi}(w)}{\partial w_{k}}=\pi_{k}-p_{k}$. We have implemented these calculations in Matlab using a modified versions of the publicly available Multi-Parametric Toolbox $(\mathrm{MPT})^{2}$. All the programs are available upon request.

\section{Conclusion}

In comparison with existing literature on the topic on multidimensional risk exposures, this work proposes a multivariate extension of the notion of comonotonicity, which involves simultaneous optimal rearrangements of two vectors of risk. With this extension, we are able to generalize Kusuoka's result and characterize subadditive, comonotonic additive and law invariant risk measures by maximal correlation functionals, which we show can be

\footnotetext{
${ }^{2} \mathrm{MPT}$ is available online at http://control.ee.ethz.ch/ mpt/.
} 
conveniently computed using optimal transportation methods. We also show that the properties of law invariance, subadditivity and comonotonic additivity can be summarized by an equivalent property, that we call strong coherence, and that we argue has a more natural economic interpretation. Further, we believe that this paper illustrates the enormous potential of the theory of optimal transportation in multivariate analysis and higher dimensional probabilities. We do not doubt that this theory will be included in the standard probabilistic toolbox in a near future.

$\dagger$ Canada Research Chair in Mathematical Economics, University of British Columbia. E-mail: ekeland@math.ubc.ca

$\S$ Corresponding author. École polytechnique, Department of Economics, 91128 Palaiseau, France. E-mail: alfred.galichon@polytechnique.edu

† Département de sciences économiques, Université de Montréal, CIRANO, CIREQ. Email: marc.henry@umontreal.ca

\section{REFERENCES}

[1] Artzner P., and F. Delbaen and J.-M. Eber and D. Heath, "Coherent measures of risk," Mathematical Finance, 9, pp. 203-228, 1999.

[2] Aurenhammer, F., Hoffmann, F., and Aronov, B., "Minkowski-type theorems and least-squares clustering," Algorithmica 20, pp. 61-76, 1998.

[3] Borwein J., and A. Lewis, Convex Analysis and Nonlinear Optimization, 2nd Edition, New York: Springer, 2006.

[4] Burgert, C., and L. Rüschendorf, "Consistent risk measures for portfolio vectors," Insurance: Mathematics and Economics 38, pp. 289-297, 2006

[5] Barrieu, P., and El Karoui, N., "Inf-convolution of risk measures and optimal risk transfer," Finance and Stochastics 9 (2), pp. 269-298, 2005.

[6] Cheridito, P. and Li, T., "Risk Measures on Orlicz Hearts," Mathematical Finance 19 2, pp. 189-214, April 2009.

[7] Dana, R.-A., "A Representation Result for Concave Schur Concave Functions," Mathematical Finance 15 (4), pp. 613-634, 2005.

[8] Delbaen, F., "Coherent risk measures on general probability spaces," Advances in Finance and Stochastics: Essays in Honour of Dieter Sondermann, pp. 1-37, Berlin: Springer, 2002. 
[9] Fabian,M., Habala, P., Hajek, P., Montesinos Santalucia, V., Pelant, J., and Zizler, V. Functional Analysis and Infinite-Dimensional Geometry, Springer: CMS Books in Mathematic, 2001

[10] Föllmer, H., and A. Schied, Stochastic Finance, de Gruyter, 2004.

[11] Frittelli, M. and Rosazza Gianin, E., "Law invariant convex risk measures," Advances in Mathematical Economics 7, pp. 33-46, 2005.

[12] Galichon, A., "The VaR at Risk," forthcoming, International Journal on Theoretical and Applied Finance.

[13] Jouini, E., Meddeb, M., and Touzi, N., "Vector valued coherent risk measures," Finance and Stochastics 4, pp. 531-552, 2004.

[14] Jouini, E., W. Schachermayer and Touzi, N., "Law invariant risk measures have the Fatou property," Advances in Mathematical Economics 9, pp. 49-71, 2006.

[15] Kusuoka, S., "On law invariant coherent risk measures," Advances in Mathematical Economics 3, pp. 83-95, 2001.

[16] Rachev, S., and Rüschendorf, L., Mass Transportation Problems. Volume I: Theory, and Volume II: Applications, New York: Springer, 1998.

[17] Rüschendorf, L., "Law invariant convex risk measures for portfolio vectors," Statistics and Decisions 24, pp. 97-108, 2006.

[18] Rüschendorf, L., "Monge - Kantorovich transportation problem and optimal couplings," Jahresbericht der DMV 3, pp. 113-137, 2007.

[19] Rüschendorf, L. and Uckelmann, L., "Numerical and analytical results for the transportation problem of Monge-Kantorovich," Metrika. International Journal for Theoretical and Applied Statistics 51, pp. $245-258,2000$.

[20] Samuelson, P., Foundations of Economic Analysis, Cambridge, MA: Harvard University Press, 1947.

[21] Schmeidler, D., "Subjective probability and expected utility without additivity," Econometrica 57, pp. 571-587, 1989.

[22] Villani, C., Topics in Optimal Transportation, Providence: American Mathematical Society, 2003.

[23] Yaari, M., "The dual theory of choice under risk," Econometrica 55, pp. 95-115, 1987.

\section{Appendix A. Illustrations}

The tâtonnement algorithm was implemented with the use of the Multi-Parametric Toolbox, and we derived the general quantile $\nabla V$ that achieves the optimal transportation of the uniform distribution on the unit cube in $\mathbb{R}^{d}$ and the empirical distribution of a sample of uniformly distributed random vectors in the unit cube in $\mathbb{R}^{d}$. The following illustrations show the Monge-Kantorovitch potential $V$, also interpreted as the buyer's indirect utility in 
our general equilibrium interpretation in the case of samples of size 7 and 27 respectively. The potential $V$ is piecewise affine, and the algorithm also requires to determine the regions over which it is affine, and their volume and center of mass. The corresponding partition is given opposite each potential plot. For illustration purposes, the dimension of the space $d$ is taken equal to 2 , but the generalized quantiles and corresponding partitions can be derived in higher dimensions.
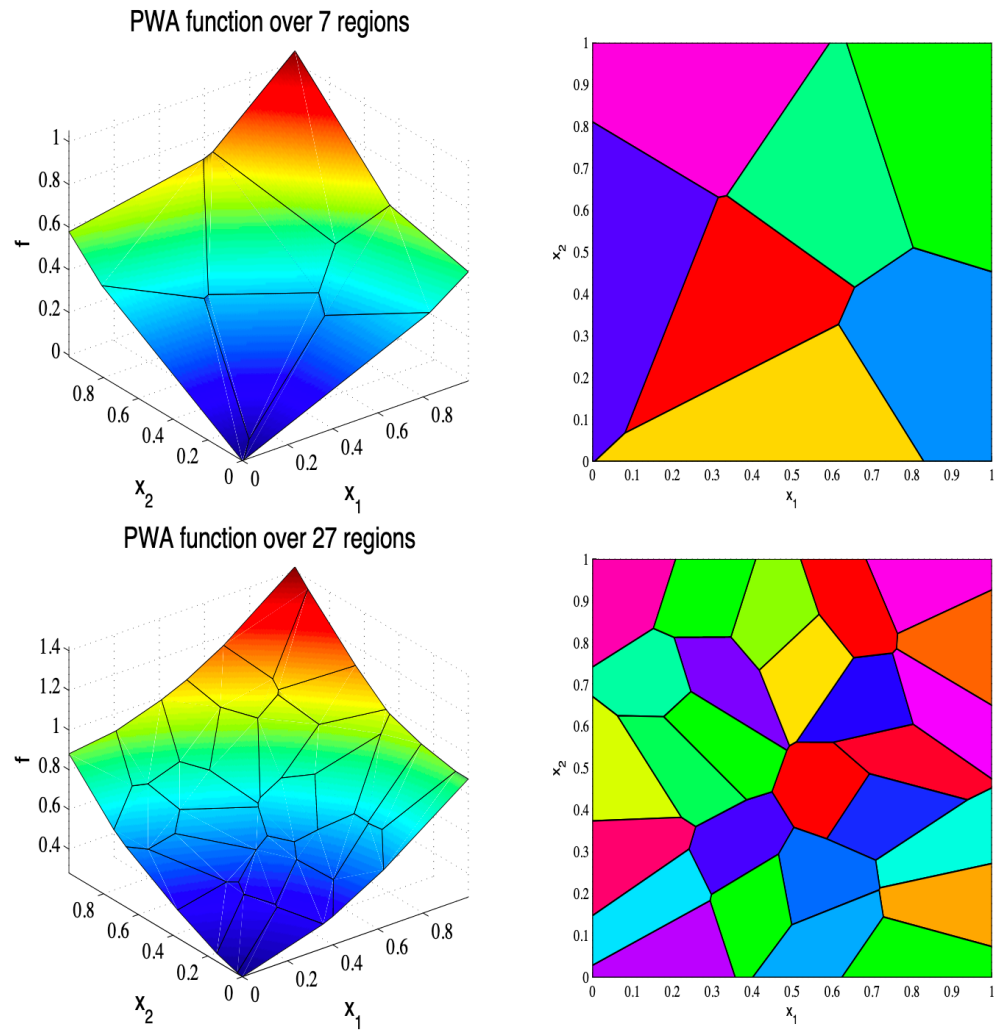

Figure 1. Mapping the uniform to a discrete discrete distribution in dimension $d=2$. Upper row: seven atom points, lower row: twenty-seven atom points. Left column: the potential $V(u)=w^{*}(u)$. Right column: the corresponding partition of the space $\mathcal{U}$. 

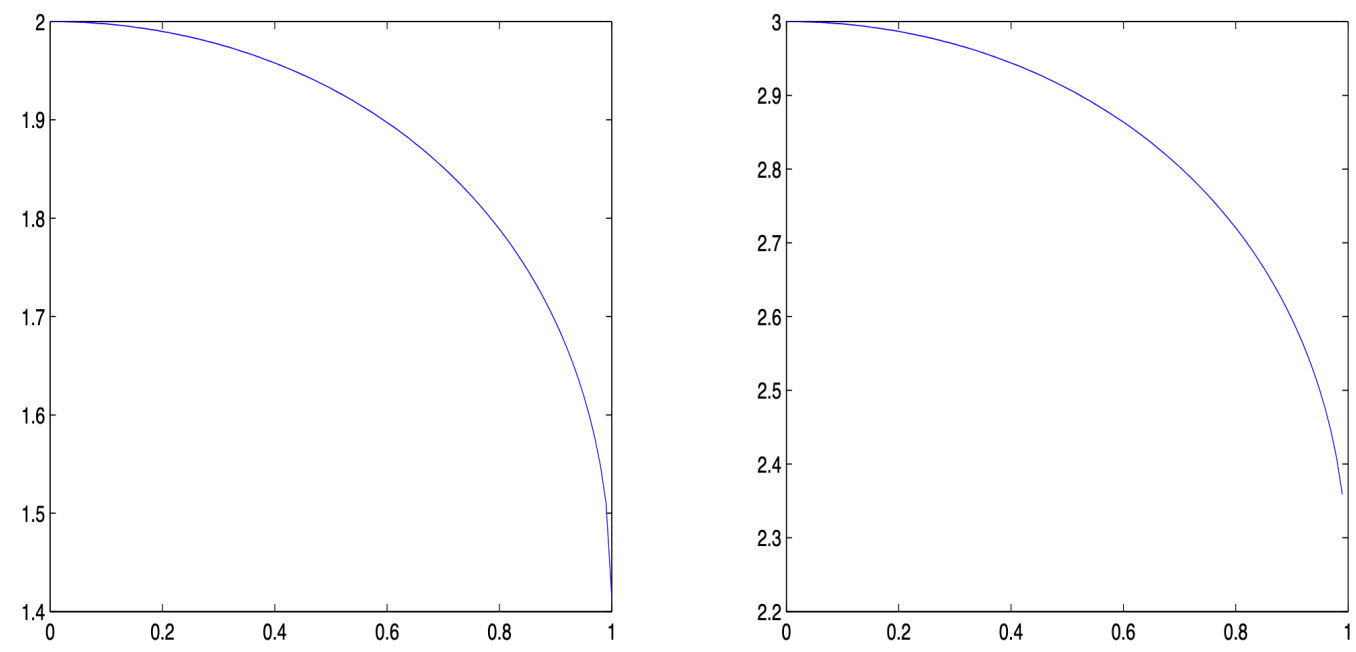

Figure 2. The value of the risk measure in the Gaussian case, plot against $\rho$. Left: $\sigma_{1}=\sigma_{2}=1$. Right: $\sigma_{1}=1, \sigma_{2}=2$.

\section{Appendix B. Results on Optimal Transportation}

In this appendix we recall basic results in Optimal Transportation theory. Roughly put, this theory characterizes the properties of the couplings of two random variables which achieve maximal correlation. We state the following basic result, due to Brenier (cf. [22], Th. 2.12, in which a proof is given).

Proposition 7. Let $\varrho$ be a maximal correlation risk measure with respect to baseline risk $U$. Then if both $\mathcal{L}_{U}$ and $\mathcal{L}_{X}$ are absolutely continuous, there exist a convex functions $V: \mathbb{R}^{d} \rightarrow \mathbb{R}$ and $W: \mathbb{R}^{d} \rightarrow \mathbb{R}$ which are Legendre-Fenchel conjugate of each other ie. 
$W=V^{*}$, and

$$
\begin{aligned}
& \varrho(X)=E[U \cdot \nabla V(U)] \\
& \varrho(X)=E[X \cdot \nabla W(X)]
\end{aligned}
$$

where the map $\nabla V$ pushes forward $\mathcal{L}_{U}$ to $\mathcal{L}_{X}$, and conversely $\nabla W$ pushes forward $\mathcal{L}_{X}$ to $\mathcal{L}_{U}$, and $\nabla W=(\nabla V)^{-1}$. When only $\mathcal{L}_{U}$ is absolutely continuous, then only those among the statements above involving $V$ alone hold, and similarly, when only $\mathcal{L}_{X}$ is absolutely continuous then only those among the statements above involving $W$ alone hold.

As $Q_{X}=\nabla V$ pushes forward measure $\mu$ on the distribution of $X$, it can be seen in some sense as a natural extension of a univariate quantile function (where $\mu=\mathcal{U}([0,1])$ - in which case $\left.Q_{X}=F_{X}^{-1}\right)$ to the multivariate setting. 